\title{
Mathematical Model of Catmull-Clark Subdivision Scheme on Regular Mesh
}

\author{
Shu-qun Liu, Bei Zhang \\ School of Computer and Communication, Lanzhou University of Technology, Lanzhou 730050, \\ China zhbeibest@163.com
}

Keywords: Subdivision, interpolation, mathematical expression, B-spline surface.

\begin{abstract}
In order to further simplify the research of limit surface properties and establish a unified mathematical model. According to Catmull-Clark subdivision method in the regular mesh at the subdivision rules, a method of calculating the mathematical expression of limit surface by interpolation was presented by using the subdivision method which is described by the curves and surfaces interpolation theory. And according to the process of solving, we prove that the limit surface which generated by the Catmull-Clark subdivision method in the regular mesh is a bi-cubic B-spline surface.
\end{abstract}

\section{Introduction}

The subdivision method is a discrete curve and surface representation based on mesh refinement, it can construct smooth surfaces from any topological mesh. The basic process is that we give a low-resolution control mesh firstly, and define the subdivision rules on the mesh, with the refinement of the mesh we can finally get a smooth surface (limit surface) [1]. Automobile, aircraft, shipbuilding and other manufacturing design, can be subdivided according to the specific subdivision rules, rapid iteration and generate the required model surface.

According to whether the limit surface is interpolated or not, the subdivision method can be divided into two types: The first kind is the approximating subdivision schemes, such as quadrilateral mesh-based Catmull-Clark subdivision method [2] and the triangular mesh-based Loop subdivision method [3]. The limit surfaces of the two subdivision methods reach $C^{1}$ continuous at extraordinary vertex and $C^{2}$ continuous at the other regular points, and the limit surface shape generated by the approximation subdivision method will shrink with respect to the initial grid. The second kind is the interpolatory subdivision schemes, which subdivides the generated limit surface into a given initial control grid vertex. Such as Dyn [4] presented a butterfly subdivision method, but the method is only applicable to the mesh within the regular points and boundary points, and its subdivision surface at the singular point can only be $C^{0}$ continuous. Due to lower continuity and restrictions on the initial control mesh, Butterfly subdivision method cannot be applied to any triangle meshes, Denis Zorin presented an modified butterfly subdivision scheme based on the Butterfly subdivision scheme to generate $C^{1}$ continuous surfaces on arbitrary triangular meshes [5]. The limit surface obtained by interpolatory subdivision schemes does not shrink, but the limit surface will produce unnecessary distortion. According to the initial control mesh, the shape of the corresponding limit surface cannot be judged well.

There is no analytic expression for the subdivision surface, so the analysis of the surface continuity and smoothness is more difficult. As the subdivision curve control polygon topology is relatively simple, therefore, the convergence and continuity of the univariate subdivision (curve) scheme has been formed into a complete framework [6-8]. For a linear static two-variable subdivision scheme, the continuity analysis is more simply when the control grid is a regular case. The local subdivision matrix can be obtained from the iterative relation, as most of the subdivision matrix has some characteristics of cyclic matrix, therefore, the Discrete Fourier Transform (DFT) can be used to simplify the solution of the eigenvalue of the matrix to reveal the convergence of the iterative process.

The remarkable characteristic of the subdivision method is that it cannot be restricted by the 
control topology mesh and can be used to shape any arbitrary topology mesh. But the existing problem is that the analysis process is complex and unfavorable to the subdivision surface properties [9-10]. Therefore, it is particularly important to find a simple and rapid method to analyze the properties of subdivision surfaces. And interpolation method is the process of finding simple function to approximately replace complex function. This paper presents a Catmull-Clark subdivision method based on the three-dimensional space curve and surface interpolation theory to describe the regular mesh, and builds the mathematical model of Catmull-Clark subdivision method based on curve and surface.

\section{Catmull-Clark Subdivision Method and Its Limit Point Formula}

Catmull and Clark extend the subdivision of bi-cubic B-spline surface into arbitrary topological structure, and their present Catmull-Clark subdivision scheme [2]. Given an initial arbitrary control-points meshes, after a Catmull-Clark subdivision, all control meshes become quadrilateral meshes. For quadrilateral control meshes, the Catmull-Clark subdivision scheme uses 1-4 quadrilateral splits to generate new mesh topologies. The subdivision rule consists of two parts: the generating of geometric point and the creation of topology.

\subsection{Geometrical Rules}

(1) New face points: the average of all of the old points defining the face. Set the vertex of the surface of $P_{0}, P_{1}, P_{2}, \cdots, P_{n}$, the new face point, is given by

$$
P_{F}=\frac{1}{n} \sum_{i=1}^{n} P_{i}
$$

On a quadrilateral mesh, is given by

$$
P_{F}=\frac{1}{4}\left(P_{1}+P_{2}+P_{3}+P_{4}\right)
$$

(2) New edge points: the average of the midpoints of the old edge with the average of the two new face points of the faces sharing the edge. Suppose that $P_{i}$ and $P_{j}$ are the two endpoints of the inner edge, the adjacent new points of the shared inner edge $P_{i} P_{j}$ are $F_{1}$ and $F_{2}$, and the new edge points is given by

$$
P_{E}=\frac{1}{4}\left(P_{i}+P_{j}+F_{1}+F_{2}\right)
$$

On the quadrilateral control mesh, assuming $P_{0}, P_{1}$ are two ends of an inner edge, $P_{0} P_{1} P_{2} P_{3}$ and $P_{0} P_{1} P_{4} P_{5}$ are two adjacent quadrilateral faces, and the new edge points is given by

$$
P_{E}=\frac{3}{8}\left(P_{0}+P_{1}\right)+\frac{1}{16}\left(P_{2}+P_{3}+P_{4}+P_{5}\right)
$$

(3) New vertex points: suppose that the vertices around the interior vertex $P$ are $P_{1}, P_{2}, P_{3}, \cdots, P_{2 i}$, where $P_{2 i-1}$ is the adjacent vertex of the vertex, $P_{2 i}$ is the diagonal vertex on the quadrilateral surface $(i=1,2,3, \cdots, n)$, and the new vertex points is given by

$$
P_{V}=\alpha_{n} P+\beta_{n} \sum_{i=1}^{n} P_{2 i}+\gamma_{n} \sum_{i=1}^{n} P_{2 i-1}
$$

Where $\alpha_{n}=1-n\left(\beta_{n}+\gamma_{n}\right), \beta_{n}=\frac{3}{2 n^{2}}, \gamma_{n}=\frac{1}{4 n^{2}}$, similar to (5), equation (5) can be converted to

$$
P_{v}=\frac{1}{n} Q+\frac{2}{n} R+\frac{n-3}{n} V
$$

Where $Q$ is the average of the vertices in the face; $R$ is the average of the midpoints of all old edges incident on the old vertex point; $P$ is old vertex point.

On the quadrilateral control grid, and the new vertex points is given by 


$$
P_{v}=\frac{1}{4} Q+\frac{1}{2} R+\frac{1}{4} V
$$

\subsection{Topological Rules}

After these points have been computed, new edges are formed by

(1) Connecting each new face point to the new edge points of the edges defining the old face.

(2) Connecting each new vertex point to the new edge points of all old edges incident on the old vertex point.

\subsection{Limit Point Formula}

Halstead[11] presented the limit point of the formula. Let $v^{(0)}$ be any vertex on initial mesh $\bar{M}^{(0)}$, which corresponds to the point at which vertex $v^{(\infty)}$ converges to the limit surface in the Catmull-Clark subdivision process. And $v^{(\infty)}$ is given by

$$
v^{(\infty)}=\frac{n^{2} v^{(0)}+4 \sum_{j} e_{j}+\sum_{j} f_{j}}{n(n+5)}
$$

Where $n$ is the degree of the vertex, $e_{j}^{(0)}$ is the adjacent point in the $v^{(0)} 1$-field, and $f_{j}^{(0)}$ is the non-adjacent point on the $v^{(0)} 1$-domain surface.

\section{Mathematical Model of Catmull-Clark Subdivision Scheme on Regular Mesh}

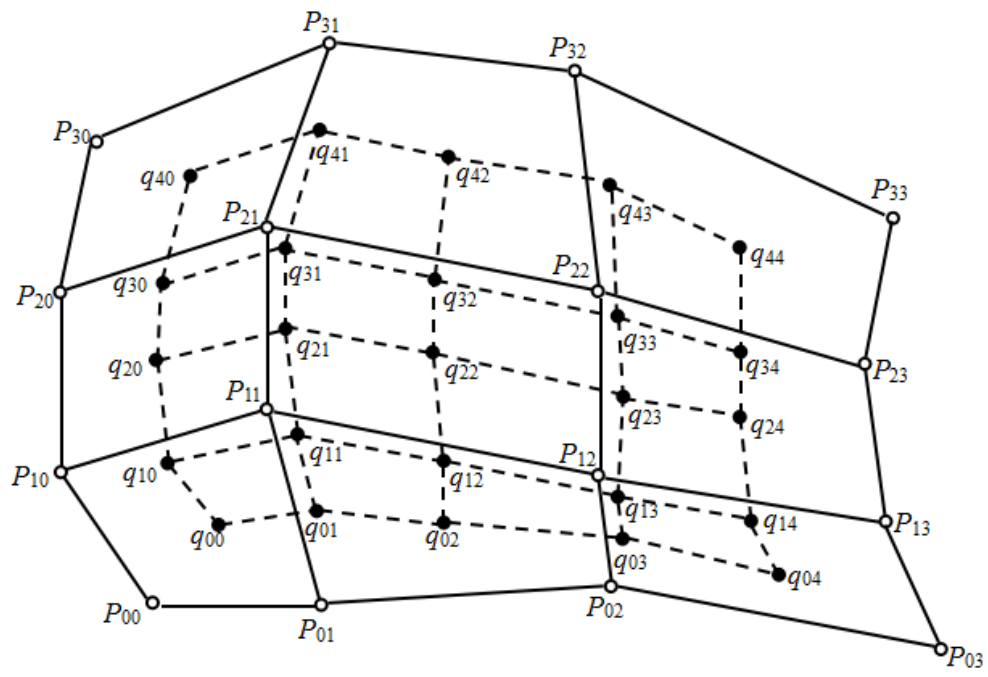

Fig. 1 Catmull-Clark subdivision scheme on regular mesh

In the regular mesh, the geometrical rules of Catmull-Clark subdivision, new face points, new edge points and new vertex generation follow (2), (3) and (7), and the subdivision is shown in Fig.1. According to Catmull-Clark subdivision rule in the regular quadrilateral mesh, the curve and surface interpolation method is used to calculate the new points. The general form of the resulting limit surface be expressed in matrix form by

$$
S(u, v)=U M P M^{T} V^{T}
$$

Where $U=\left(\begin{array}{llll}u^{3} & u^{2} & u & 1\end{array}\right), V=\left(\begin{array}{llll}v^{3} & v^{2} & v & 1\end{array}\right)$ are the parameter vectors, and

$$
P=\left(\begin{array}{llll}
P_{00} & P_{10} & P_{20} & P_{30} \\
P_{01} & P_{11} & P_{21} & P_{31} \\
P_{02} & P_{12} & P_{22} & P_{32} \\
P_{03} & P_{13} & P_{23} & P_{33}
\end{array}\right)
$$

is the set of control points, which are arranged on a topologically quadrilateral mesh according to their subscripts, and 
is the matrix to be solved.

$$
M=\left(\begin{array}{llll}
a_{0} & a_{1} & a_{2} & a_{3} \\
b_{0} & b_{1} & b_{2} & b_{3} \\
c_{0} & c_{1} & c_{2} & c_{3} \\
d_{0} & d_{1} & d_{2} & d_{3}
\end{array}\right)
$$

De-Boor algorithm is mainly used to generate continuous curves from discrete points according to algorithm rules, and extend the algorithm to 3D space curves and surfaces, and get generalized De-Boor algorithm to generate space curves and surfaces. In mathematics, the method of generating curves and surfaces conforms to the idea of interpolation, so it can be regarded as the interpolation scheme, according to the curve and surface interpolation theory, combined with subdivision rules, set the parameter values, there,

$$
\begin{aligned}
S(0,0)= & \frac{1}{6}\left(\frac{1}{6} P_{00}+\frac{2}{3} P_{01}+\frac{1}{6} P_{02}\right)+\frac{2}{3}\left(\frac{1}{6} P_{10}+\frac{2}{3} P_{11}+\frac{1}{6} P_{12}\right)+\frac{1}{6}\left(\frac{1}{6} P_{20}+\frac{2}{3} P_{21}+\frac{1}{6} P_{22}\right) \\
S(0,1)= & \frac{1}{6}\left(\frac{1}{6} P_{10}+\frac{2}{3} P_{11}+\frac{1}{6} P_{12}\right)+\frac{2}{3}\left(\frac{1}{6} P_{20}+\frac{2}{3} P_{21}+\frac{1}{6} P_{22}\right)+\frac{1}{6}\left(\frac{1}{6} P_{30}+\frac{2}{3} P_{31}+\frac{1}{6} P_{32}\right) \\
S(1,0)= & \frac{1}{6}\left(\frac{1}{6} P_{01}+\frac{2}{3} P_{02}+\frac{1}{6} P_{03}\right)+\frac{2}{3}\left(\frac{1}{6} P_{11}+\frac{2}{3} P_{12}+\frac{1}{6} P_{13}\right)+\frac{1}{6}\left(\frac{1}{6} P_{21}+\frac{2}{3} P_{22}+\frac{1}{6} P_{23}\right) \\
S(1,1)= & \frac{1}{6}\left(\frac{1}{6} P_{11}+\frac{2}{3} P_{12}+\frac{1}{6} P_{13}\right)+\frac{2}{3}\left(\frac{1}{6} P_{21}+\frac{2}{3} P_{22}+\frac{1}{6} P_{23}\right)+\frac{1}{6}\left(\frac{1}{6} P_{31}+\frac{2}{3} P_{32}+\frac{1}{6} P_{33}\right) \\
S\left(0, \frac{1}{2}\right) & =\frac{1}{48}\left(\frac{1}{6} P_{00}+\frac{2}{3} P_{01}+\frac{1}{6} P_{02}\right)+\frac{23}{48}\left(\frac{1}{6} P_{10}+\frac{2}{3} P_{11}+\frac{1}{6} P_{12}\right) \\
& +\frac{23}{48}\left(\frac{1}{6} P_{20}+\frac{2}{3} P_{21}+\frac{1}{6} P_{22}\right)+\frac{1}{48}\left(\frac{1}{6} P_{30}+\frac{2}{3} P_{31}+\frac{1}{6} P_{32}\right) \\
S\left(0, \frac{1}{3}\right) & =\frac{4}{81}\left(\frac{1}{6} P_{00}+\frac{2}{3} P_{01}+\frac{1}{6} P_{02}\right)+\frac{31}{54}\left(\frac{1}{6} P_{10}+\frac{2}{3} P_{11}+\frac{1}{6} P_{12}\right) \\
& +\frac{10}{27}\left(\frac{1}{6} P_{20}+\frac{2}{3} P_{21}+\frac{1}{6} P_{22}\right)+\frac{1}{162}\left(\frac{1}{6} P_{30}+\frac{2}{3} P_{31}+\frac{1}{6} P_{32}\right)
\end{aligned}
$$

According to the general form of the initial limit surface, bring in the parameter values set in (9), the points are given by

$$
\begin{aligned}
& S(0,0)=\left(d_{0} P_{00}+d_{1} P_{01}+d_{2} P_{02}+d_{3} P_{03}\right) d_{0}+\left(d_{1} P_{00}+d_{1} P_{11}+d_{2} P_{12}+d_{3} P_{13}\right) d_{1} \\
& +\left(d_{0} P_{20}+d_{1} P_{21}+d_{2} P_{22}+d_{3} P_{23}\right) d_{2}+\left(d_{0} P_{30}+d_{1} P_{31}+d_{2} P_{32}+d_{3} P_{33}\right) d_{3} \\
& =\frac{1}{6}\left(\frac{1}{6} P_{00}+\frac{2}{3} P_{01}+\frac{1}{6} P_{02}\right)+\frac{2}{3}\left(\frac{1}{6} P_{10}+\frac{2}{3} P_{11}+\frac{1}{6} P_{12}\right)+\frac{1}{6}\left(\frac{1}{6} P_{20}+\frac{2}{3} P_{21}+\frac{1}{6} P_{22}\right) \\
& S(0,1)=\left(d_{0} P_{00}+d_{1} P_{01}+d_{2} P_{02}+d_{3} P_{03}\right)\left(a_{0}+b_{0}+c_{0}+d_{0}\right) \\
& +\left(d_{0} P_{10}+d_{1} P_{11}+d_{2} P_{12}+d_{3} P_{13}\right)\left(a_{1}+b_{1}+c_{1}+d_{1}\right) \\
& +\left(d_{0} P_{20}+d_{1} P_{21}+d_{2} P_{22}+d_{3} P_{23}\right)\left(a_{2}+b_{2}+c_{2}+d_{2}\right) \\
& +\left(d_{0} P_{30}+d_{1} P_{31}+d_{2} P_{32}+d_{3} P_{33}\right)\left(a_{3}+b_{3}+c_{3}+d_{3}\right) \\
& = \\
& \quad \frac{1}{6}\left(\frac{1}{6} P_{10}+\frac{2}{3} P_{11}+\frac{1}{6} P_{12}\right)+\frac{2}{3}\left(\frac{1}{6} P_{20}+\frac{2}{3} P_{21}+\frac{1}{6} P_{22}\right) \\
& \\
& +\frac{1}{6}\left(\frac{1}{6} P_{30}+\frac{2}{3} P_{31}+\frac{1}{6} P_{32}\right)
\end{aligned}
$$




$$
\begin{aligned}
S\left(0, \frac{1}{2}\right)= & \left(d_{0} P_{00}+d_{1} P_{01}+d_{2} P_{02}+d_{3} P_{03}\right)\left(\frac{a_{0}}{8}+\frac{b_{0}}{4}+\frac{c_{0}}{2}+d_{0}\right) \\
& +\left(d_{0} P_{10}+d_{1} P_{11}+d_{2} P_{12}+d_{3} P_{13}\right)\left(\frac{a_{1}}{8}+\frac{b_{1}}{4}+\frac{c_{1}}{2}+d_{1}\right) \\
& +\left(d_{0} P_{20}+d_{1} P_{21}+d_{2} P_{22}+d_{3} P_{23}\right)\left(\frac{a_{2}}{8}+\frac{b_{2}}{4}+\frac{c_{2}}{2}+d_{2}\right) \\
& +\left(d_{0} P_{30}+d_{1} P_{31}+d_{2} P_{32}+d_{3} P_{33}\right)\left(\frac{a_{3}}{8}+\frac{b_{3}}{4}+\frac{c_{3}}{2}+d_{3}\right) \\
= & \frac{1}{48}\left(\frac{1}{6} P_{00}+\frac{2}{3} P_{01}+\frac{1}{6} P_{02}\right)+\frac{23}{48}\left(\frac{1}{6} P_{10}+\frac{2}{3} P_{11}+\frac{1}{6} P_{12}\right) \\
& +\frac{23}{48}\left(\frac{1}{6} P_{20}+\frac{2}{3} P_{21}+\frac{1}{6} P_{22}\right)+\frac{1}{48}\left(\frac{1}{6} P_{30}+\frac{2}{3} P_{31}+\frac{1}{6} P_{32}\right) \\
S\left(0, \frac{1}{3}\right)= & \left(d_{0} P_{00}+d_{1} P_{01}+d_{2} P_{02}+d_{3} P_{03}\right)\left(\frac{a_{0}}{27}+\frac{b_{0}}{9}+\frac{c_{0}}{3}+d_{0}\right) \\
& +\left(d_{0} P_{10}+d_{1} P_{11}+d_{2} P_{12}+d_{3} P_{13}\right)\left(\frac{a_{1}}{27}+\frac{b_{1}}{9}+\frac{c_{1}}{3}+d_{1}\right) \\
& +\left(d_{0} P_{20}+d_{1} P_{21}+d_{2} P_{22}+d_{3} P_{23}\right)\left(\frac{a_{2}}{27}+\frac{b_{2}}{9}+\frac{c_{2}}{3}+d_{2}\right) \\
& +\left(d_{0} P_{30}+d_{1} P_{31}+d_{2} P_{32}+d_{3} P_{33}\right)\left(\frac{a_{3}}{27}+\frac{b_{3}}{9}+\frac{c_{3}}{3}+d_{3}\right) \\
= & \frac{4}{81}\left(\frac{1}{6} P_{00}+\frac{2}{3} P_{01}+\frac{1}{6} P_{02}\right)+\frac{31}{54}\left(\frac{1}{6} P_{10}+\frac{2}{3} P_{11}+\frac{1}{6} P_{12}\right) \\
& +\frac{10}{27}\left(\frac{1}{6} P_{20}+\frac{2}{3} P_{21}+\frac{1}{6} P_{22}\right)+\frac{1}{162}\left(\frac{1}{6} P_{30}+\frac{2}{3} P_{31}+\frac{1}{6} P_{32}\right)
\end{aligned}
$$

For all equations in (16), (17), (18) and (19) to be constant, their corresponding coefficients should be equal, there

$$
\begin{aligned}
& \left\{\begin{array}{l}
d_{0}^{2}=\frac{1}{36} \\
d_{1}^{2}=\frac{4}{9} \\
d_{2}^{2}=\frac{1}{36} \\
d_{3}^{2}=0
\end{array}\right. \\
& \left\{\begin{array}{l}
d_{0}\left(\frac{a_{0}}{8}+\frac{b_{0}}{4}+\frac{c_{0}}{2}+d_{0}\right)=\frac{1}{288} \\
d_{0}\left(\frac{a_{1}}{8}+\frac{b_{1}}{4}+\frac{c_{1}}{2}+d_{1}\right)=\frac{23}{288} \\
d_{0}\left(\frac{a_{2}}{8}+\frac{b_{2}}{4}+\frac{c_{2}}{2}+d_{2}\right)=\frac{23}{288} \\
d_{0}\left(\frac{a_{3}}{8}+\frac{b_{3}}{4}+\frac{c_{3}}{2}+d_{3}\right)=\frac{1}{288} \\
d_{0}\left(a_{2}+b_{2}+c_{2}+d_{2}\right)=\frac{1}{9} \\
d_{0}\left(a_{3}+b_{3}+c_{3}+d_{3}\right)=\frac{1}{36}
\end{array}\right. \\
& d_{0}\left(a_{1}+b_{1}+c_{1}+d_{1}\right)=\frac{1}{36} \\
& \left.d_{0}\left(\frac{a_{1}}{27}+\frac{b_{1}}{9}+\frac{c_{1}}{3}+d_{1}\right)=\frac{a_{2}}{27}+\frac{b_{2}}{9}+\frac{c_{2}}{3}+d_{2}\right)=\frac{10}{162} \\
& d_{0}\left(\frac{a_{3}}{27}+\frac{b_{3}}{9}+\frac{c_{3}}{3}+d_{3}\right)=\frac{1}{972}
\end{aligned}
$$

Combining the four equations in (20), and solving equations, then there 


$$
\left\{\begin{array} { l } 
{ d _ { 0 } = \frac { 1 } { 6 } } \\
{ d _ { 1 } = \frac { 2 } { 3 } } \\
{ d _ { 2 } = \frac { 1 } { 6 } } \\
{ d _ { 3 } = 0 }
\end{array} \quad \left\{\begin{array} { l } 
{ a _ { 0 } = - \frac { 1 } { 6 } } \\
{ a _ { 1 } = \frac { 1 } { 2 } } \\
{ a _ { 2 } = - \frac { 1 } { 2 } } \\
{ a _ { 3 } = \frac { 1 } { 6 } }
\end{array} \quad \left\{\begin{array} { l } 
{ b _ { 0 } = \frac { 1 } { 2 } } \\
{ b _ { 1 } = - 1 } \\
{ b _ { 2 } = \frac { 1 } { 2 } } \\
{ b _ { 3 } = 0 }
\end{array} \quad \left\{\begin{array}{l}
c_{0}=-\frac{1}{2} \\
c_{1}=0 \\
c_{2}=\frac{1}{2} \\
c_{3}=0
\end{array}\right.\right.\right.\right.
$$

Another set of solutions of the system does not meet the subdivision rules of the Catmull-Clark curve subdivision, so it does not conform to the subdivision rule of the Catmull-Clark subdivision on the surface. Then there

$$
M=\left(\begin{array}{llll}
a_{0} & a_{1} & a_{2} & a_{3} \\
b_{0} & b_{1} & b_{2} & b_{3} \\
c_{0} & c_{1} & c_{2} & c_{3} \\
d_{0} & d_{1} & d_{2} & d_{3}
\end{array}\right)=\left(\begin{array}{rrrr}
-\frac{1}{6} & \frac{1}{2} & -\frac{1}{2} & \frac{1}{6} \\
\frac{1}{2} & -1 & \frac{1}{2} & 0 \\
-\frac{1}{2} & 0 & \frac{1}{2} & 0 \\
\frac{1}{6} & \frac{2}{3} & \frac{1}{6} & 0
\end{array}\right)=\frac{1}{6}\left(\begin{array}{rrrr}
-1 & 3 & -3 & 1 \\
3 & -6 & 3 & 0 \\
-3 & 0 & 3 & 0 \\
1 & 4 & 1 & 0
\end{array}\right)
$$

The mathematical expression of the limit surface generated by the Catmull-Clark subdivision method on the regular mesh is given by

$$
\begin{gathered}
\left(\begin{array}{llll}
u^{3} & u^{2} & u & 1
\end{array}\right)\left(\begin{array}{rrrr}
-1 & 3 & -3 & 1 \\
3 & -6 & 3 & 0 \\
-3 & 0 & 3 & 0 \\
1 & 4 & 1 & 0
\end{array}\right)\left(\begin{array}{llll}
P_{00} & P_{10} & P_{20} & P_{30} \\
P_{01} & P_{11} & P_{21} & P_{31} \\
P_{02} & P_{12} & P_{22} & P_{32} \\
P_{03} & P_{13} & P_{23} & P_{33}
\end{array}\right)\left(\begin{array}{rrrr}
-1 & 3 & -3 & 1 \\
3 & -6 & 0 & 4 \\
-3 & 3 & 3 & 1 \\
1 & 0 & 0 & 0
\end{array}\right)\left(\begin{array}{c}
v^{3} \\
v^{2} \\
v \\
1
\end{array}\right) \\
36
\end{gathered}
$$

In the quadrilateral initial control grid of Fig. 1, using the limit point formula (8), the corresponding limit point position on the limit surface is:

$$
\begin{aligned}
& P_{11}^{(\infty)}=\frac{16 P_{11}+4\left(P_{21}+P_{12}+P_{01}+P_{10}\right)+\left(P_{00}+P_{20}+P_{22}+P_{02}\right)}{36} \\
& P_{21}^{(\infty)}=\frac{16 P_{21}+4\left(P_{20}+P_{11}+P_{22}+P_{31}\right)+\left(P_{30}+P_{32}+P_{12}+P_{10}\right)}{36} \\
& P_{12}^{(\infty)}=\frac{16 P_{12}+4\left(P_{11}+P_{22}+P_{13}+P_{02}\right)+\left(P_{21}+P_{23}+P_{13}+P_{01}\right)}{36} \\
& P_{22}^{(\infty)}=\frac{16 P_{22}+4\left(P_{21}+P_{12}+P_{23}+P_{32}\right)+\left(P_{31}+P_{33}+P_{11}+P_{13}\right)}{36}
\end{aligned}
$$

After simplification (10), (11), (12), (13) ,with the formula (24), (25), (26) , (27) comparison, then there

$$
S(0,0)=P_{11}^{(\infty)}, \quad S(0,1)=P_{21}^{(\infty)}, \quad S(1,0)=P_{12}^{(\infty)}, S(1,1)=P_{22}^{(\infty)}
$$

The subdivision model of the Catmull-Clark subdivision method in the regular mesh is established, and the resulting limit surface is a bi-cubic B-spline surface. From the established mathematical model, it is obvious that the properties of the convergence, differentiability and curvature of the subdivision surface can be directly studied. 


\section{Conclusions}

According to the subdivision rule of the Catmull-Clark subdivision method in the regular mesh we describe the subdivision rule by the 3D space surface interpolation theory, then the mathematic expression of the limit surface is obtained by using undetermined coefficient method, and the mathematic model of the subdivision method is established. The results show that the limit surface generated by the Catmull-Clark subdivision method in the regular mesh is a tensor product bi-cubic B-spline surface [12].

Using mathematical analysis, the mathematic model established above can be used to study the properties of subdivision surfaces, such as convergence, continuity, differentiability, and so on, and the guide vector and curvature of the surface also can be obtained. Using the discrete Fourier transform to analyze the properties of limit surfaces is more simply than using local subdivision matrices.

In this paper, only the mathematical model of Catmull-Clark subdivision method in the regular mesh is established, and the initial control mesh is not considered. So we can focus on the mathematical model of limit surface at irregular mesh.

\section{References}

[1] Schröder P, Zorin D, DeRose T, et al. Subdivision for modeling and animation[J]. ACM SIGGRAPH Course Notes, 1998, 12.

[2] Catmull E, Clark J. Recursively generated B-spline surfaces on arbitrary topological meshes[J]. Computer-aided design, 1978, 10(6): 350-355.

[3] Loop C. Smooth subdivision surfaces based on triangles[D]. [Master's thesis]. Salt Lake City: Department of Mathematics University of Utah, 1987.

[4] Dyn N, Levine D, Gregory J A. A butterfly subdivision scheme for surface interpolation with tension control[J]. ACM transactions on Graphics (TOG), 1990, 9(2): 160-169.

[5] Zorin D, Schröder P, Sweldens W. Interpolating subdivision for meshes with arbitrary topology[C]//Proceedings of the 23rd annual conference on Computer graphics and interactive techniques. ACM, 1996: 189-192

[6] Cavaretta A S, Dahmen W, Micchelli C A. Stationary subdivision[M]. American Mathematical Soc., 1991 , 93(453):186.

[7] Micchelli C A. Mathematical Aspects of Geometric Modeling[M]// Mathematical aspects of geometric modeling. Society for Industrial and Applied Mathematics, 1995.

[8] Warren J, Weimer H. Subdivision methods for geometric design: A constructive approach[M]. Morgan Kaufmann, 2001:276-285.

[9] Stam J. Exact evaluation of Catmull-Clark subdivision surfaces at arbitrary parameter values[C]//Proceedings of the 25th annual conference on Computer graphics and interactive techniques. ACM, 1998: 395-404.

[10] Stam J. Evaluation of Loop subdivision surfaces [C]//Proceedings of the 25th annual conference on Computer graphics and interactive techniques. ACM, 1998: 405-417.

[11] Halstead M, Kass M, DeRose T. Efficient, fair interpolation using Catmull-Clark surfaces[C]//Proceedings of the 20th annual conference on Computer graphics and interactive techniques. ACM, 1993: 35-44.

[12] CHEN Wei, WANG Rong-Qing, et al. Weighted Catmull-Clark Surface[J]. Journal of Computer- Aided Design \& Computer Graphics 2004, 12(4): 277-280. 